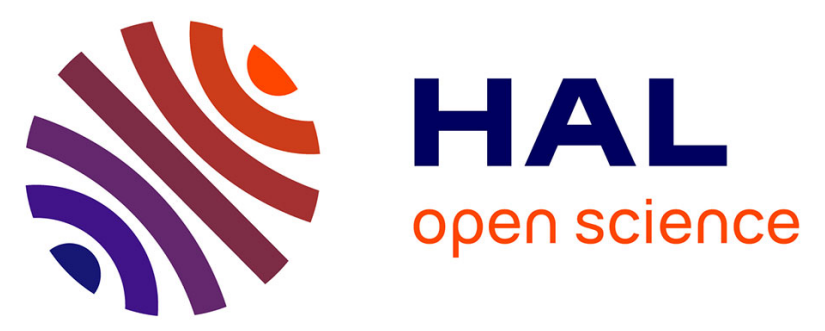

\title{
TrafficGen: A Flexible Tool For Informing Agent-Based Traffic Simulations With Open Data
}

\author{
Alexandre Bonhomme, Philippe Mathieu, Sébastien Picault
}

\section{To cite this version:}

Alexandre Bonhomme, Philippe Mathieu, Sébastien Picault. TrafficGen: A Flexible Tool For Informing Agent-Based Traffic Simulations With Open Data. 13th International Conference on Practical Applications of Agents and Multi-Agent Systems (PAAMS), Jun 2015, Salamanca, Spain. pp.259262, 10.1007/978-3-319-18944-4_22. hal-01159981

\section{HAL Id: hal-01159981 \\ https://inria.hal.science/hal-01159981}

Submitted on 15 Feb 2016

HAL is a multi-disciplinary open access archive for the deposit and dissemination of scientific research documents, whether they are published or not. The documents may come from teaching and research institutions in France or abroad, or from public or private research centers.
L'archive ouverte pluridisciplinaire HAL, est destinée au dépôt et à la diffusion de documents scientifiques de niveau recherche, publiés ou non, émanant des établissements d'enseignement et de recherche français ou étrangers, des laboratoires publics ou privés. 


\title{
TrafficGen: a flexible tool for informing agent-based traffic simulations with open data
}

\author{
Alexandre Bonhomme, Philippe Mathieu, and Sébastien Picault \\ Multiagent team, CRIStAL UMR CNRS 9189 \\ University of Lille - Science and Technology \\ Villeneuve d'Ascq, France \\ \{philippe.mathieu, sebastien.picault\}@univ-lille1.fr
}

\begin{abstract}
Multi-Agent Systems have been widely used for traffic simulation. The modeling of individuals allows indeed to introduce a behavioral diversity which is crucial to obtain realistic simulation outcomes. The recent growth of open geographical databases and related flow information provides an opportunity for enhancing traffic simulators with data automatically retrieved from the real world and updated regularly. We present here TrafficGen, a highly modular platform based on the integration of such open data within a library of rule-based behaviors, in order to provide a versatile decision support tool in traffic.
\end{abstract}

Keywords: Multi-Agent Simulation; GIS; Road Traffic; Traffic Generator; Decision Support System

\section{Introduction}

Individual-based approaches proved very early their benefits in traffic simulation $[1,2]$, especially to assess the outcome of individual decisions at a macroscopic scale. But the diversity of goals of such simulations, from flow prediction to immersive simulation to study car ergonomics, either in academic or industrial contexts, lead to the development of dedicated tools with a lack of reusability and adaptability. Yet, there is a growing need for simulation tools able to handle and compare various scenarios and hypotheses, so as to support decision in traffic management or public policies. More recently, the use of statistical data to tune the behaviors of driver agents has also become a growing issue [3,4]. The TrafficGen project, which is demonstrated here, is meant as an answer to this problematic by studying how to combine flexibility in behavior design and model revision, together with the use of geographical data and flow information in several open formats.

\section{Main purpose}

Modular and flexible behavior modeling. In most traffic simulators, agents are in general limited to vehicles: cars, bicycles, buses, etc. and by extension, pedestrians. Those agents are in charge of modeling all the traffic complexity through 
their behaviors, which are tightly dependent on the aims of the simulator. But, in order to test and compare various scenarios or assumptions, e.g. regarding intermodality, or drastic changes in transport policies, the key problem is rather to endow the user with the capability of accessing explicitly (and intelligibly) the behavioral models that reflect those assumptions, choosing those to use in a scenario, and easily redesigning the experiments, without being forced to rewrite any line of code.

To do so, we rely upon the "Interaction-Oriented Approach" (IODA) which handles each entity involved in the system as an agent [5] endowed with capabilites of interacting with the others [6]. Interactions are abstract condition/action rules which constitute the declarative part of the models, and are designed independently from the agents. Hence, they offer a modular and reusable way to build and test the behaviors of all agents in various contexts. Driver habits, path planning, scripted routes, environment learning, can be modeled through this unified, rule-based approach, and combined to build arbitrarily complex vehicle behaviors.

Exploitation of open data. Besides, we aim at using the growing amount of open data available both in geographical information systems and in transport, in order to enhance the realism of simulations. Regarding traffic path information, we tackled two formats: OSM, used in the OpenStreetMap participatory project ${ }^{1}$ which provides exhaustive cartographic information, and OpenDrive ${ }^{2}$, designed for describing road features.

Depending on the purpose of each experiment, data related to an interest area are retrieved, then filtered to keep only relevant information (e.g. highways, cycle lanes, or tram lines) which is also mapped to the corresponding kinds of vehicles. Besides, special transport items such as roads, crossroads, traffic lights, bus stops, tram stations, etc. are also represented by agents according to the IODA approach, so that they are endowed with a behavior (even simple) which can be modified interactively during the simulation (e.g. during market hours some roads are closed). This allows to reduce the complexity of driver agents by delegating part of their behaviors to infrastructure agents, in a way close to the affordances theory [7]. In order to generate realistic populations of vehicles, generator agents can be placed on ways and parameterized either through probability laws or real data. Finally, additional agents measure the characteristics of simulated vehicles (probes) so as to bridge the gap with the macroscopic level, or introduce specific events (such as a local speed reduction in response of pollution peaks) [8].

\section{Demonstration}

The TrafficGen platform demonstrated here has been built as a proof of concept in order to asses our approach (and not currently for competing in performances

\footnotetext{
${ }^{1}$ http://wwww .openstreetmap.org

${ }^{2}$ http://www.opendrive.org
} 
with industrial products). In this demonstration, we can select any interest area from OpenStreetMap, apply the filtering to select part of the available ways within a few seconds, and run the simulation (see fig. 1). We give examples of interactive experiments that can be carried out (road closing, local speed reduction, etc.) and of the corresponding measurements. The level of detail can be tuned according to the needs (fig. 2), and depending on the aims of the experiments other transport modes can be studied within the same platform (e.g. metro, fig. 3), by changing the filter.
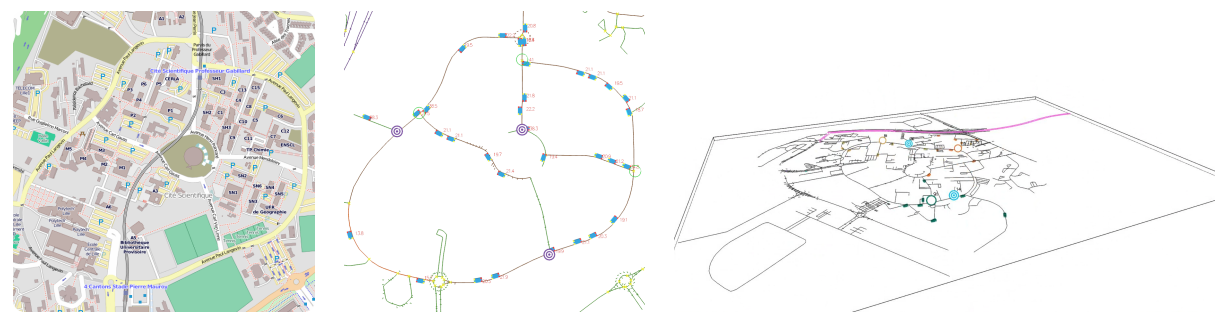

Fig. 1. From an extraction of OSM data (left), the relevant ways are imported in TrafficGen (middle) for building the appropriate environment for the simulation (right).
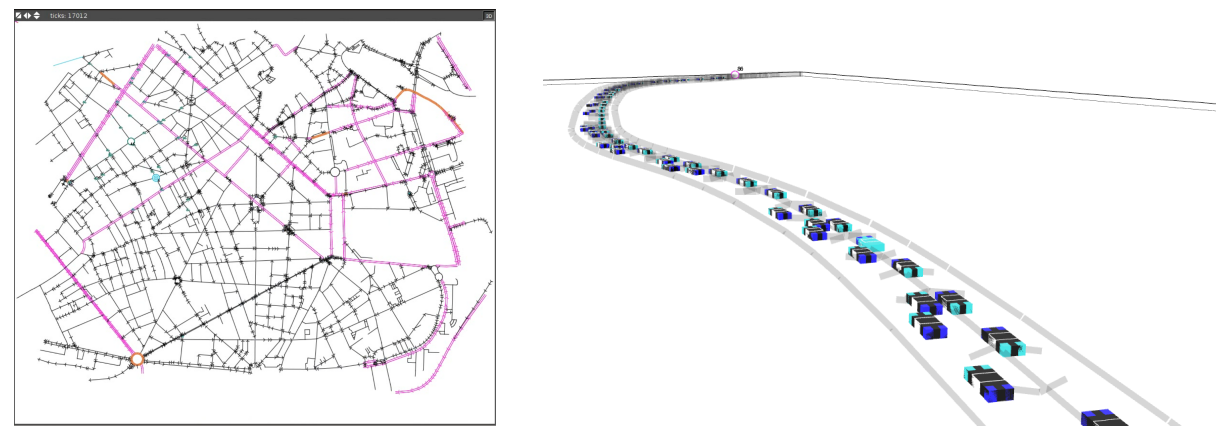

Fig. 2. Left: part of the city center of Lille (France) loaded in TrafficGen; right: Traffic on French highway A23.

\section{Conclusion}

We have presented and demonstrated the TrafficGen platform, aimed at providing a multi-purpose approach to traffic simulation, by allowing the user to easily compare scenarios and hypotheses within actual geographical data and flow information. This tool has proven its efficiency to model road traffic (fig. 1, 

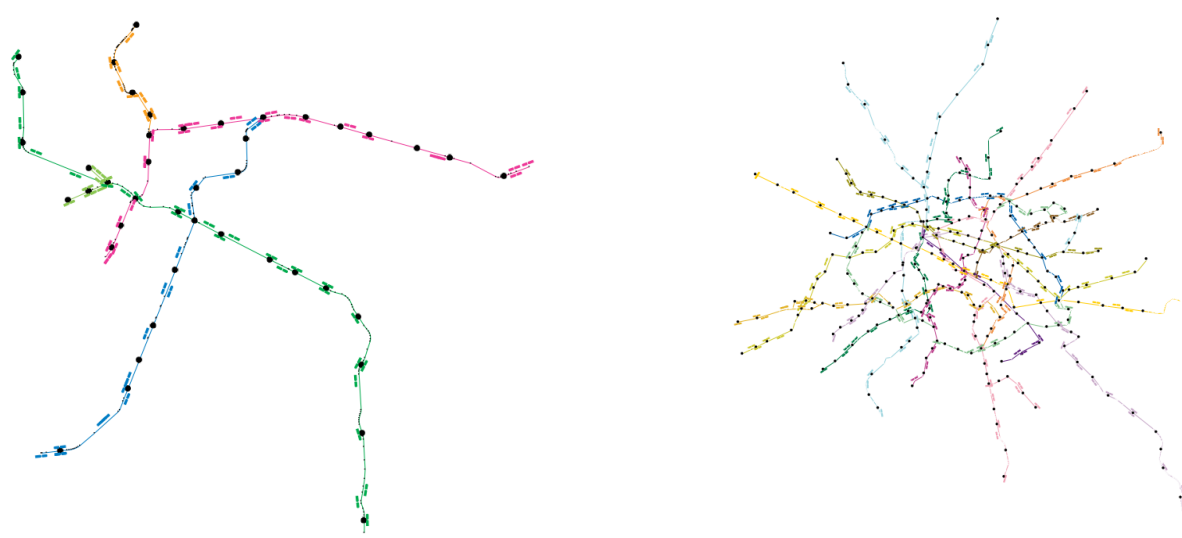

Fig. 3. A simulation of the subways of Lyon (left) and Paris (right) in TrafficGen.

2) as well as rail (fig. 3). Ongoing work on this project focus, on the one hand, on concrete case studies in the city of Lille (France) based on recorded traffic measures, and on the other hand, the extension of our model towards multi-level simulation, grounded on our previous work in this domain [9].

\section{References}

1. Espié, S., Saad, F., Schnetzler, B., Bourlier, F., Djemane, N.: Microscopic traffic simulation and driver behaviour modelling: the ARCHISIM project. In: Conf. Road Safety in Europe and Strategic Highway Research Program (SHRP). (1994) 22-31

2. Champion, A., Mandiau, R., Kolski, C., Heidet, A., Kemeny, A.: Traffic generation with the SCANeR ${ }^{\mathrm{TM}}$ simulator: towards a multi-agent architecture. In: Driving Simulation Conference. (1999) 311-324

3. Lacroix, B., Mathieu, P., Kemeny, A.: Formalizing the construction of populations in multi-agent simulations. J. Eng. App. of AI 26(1) (2013) 211-226

4. Darty, K., Saunier, J., Sabouret, N.: A method for semi-automatic explicitation of agent's behavior: application to the study of an immersive driving simulator. In: 6th Int. Conf. on Agents and AI (ICAART), SciTePress (2014) 81-91

5. Kubera, Y., Mathieu, P., Picault, S.: Everything can be agent! In: 9th Int. Joint Conf. on Auton. Agents and Multi-Agent Systems (AAMAS), IFAAMAS (2010) $1547-1548$

6. Kubera, Y., Mathieu, P., Picault, S.: IODA: An interaction-oriented approach for multi-agent based simulations. J. of Auton. Agents and Multi-Agent Systems 23(3) (2011) 303-343

7. Gibson, J.: The Ecological Approach to Visual Perception. Hillsdale (1979)

8. Bonhomme, A., Mathieu, P., Picault, S.: A versatile description framework for modeling behaviors in traffic simulations. In: Proc. of the IEEE 26th Int. Conf. on Tools with AI (ICTAI), IEEE Press (2014) 937-944

9. Picault, S., Mathieu, P.: An interaction-oriented model for multi-scale simulation. In: Proc. of the 22nd Int. Joint Conf. on AI (IJCAI), AAAI (2011) 332-337 